\title{
The heat flux near the contact line of the droplets on heated foil
}

\author{
Vyacheslav Cheverda ${ }^{1,4, a}$, Andrey Karchevsky ${ }^{2,3}$ \\ ${ }^{1}$ Kutateladze Institute of Thermophysics SB RAS, Novosibirsk 630090, Russia \\ ${ }^{2}$ Novosibirsk State University, Novosibirsk 630090, Russia \\ ${ }^{3}$ Sobolev Institute of Mathematics SB RAS, Novosibirsk 630090, Russia \\ ${ }^{4}$ Tomsk Polytechnic University, Tomsk 634050, Russia
}

\begin{abstract}
The heat flux distribution near the contact lines of one or several (3) drops on a horizontal heated constantan foil is investigated. The temperature distribution of the bottom foil surface was measured by the FLIR infrared (IR) camera. To measure the heat flux distribution near the contact lines numerical solution of the Cauchy problem is used. The maximum of the heat flux density is observed near the contact line and it exceeds the average heat flux density by several times. This is due to relatively high heat conductivity coefficient of the foil material and high evaporation rate in the contact line region, so the heat flux from the foil periphery (out of the drop) is enter to the contact line region of the drop.
\end{abstract}

\section{Introduction}

Liquid droplets on the substrate are widely distributed in nature, techniques, and technology. The wetting angle is a fundamental macroscopic characteristic of the contact line. The wetting (contact) angle is determined by the Young equation, [1]. Analysis of papers where drop is placed on the heated substrate with different level of gravity shows that there is not enough information about the process of evaporation and wetting of a solid substrate for variable levels of gravity [2, 3]. The process in a sessile liquid drop on the heated substrates with different wettability are investigated numerically and experimentally in $[4,5]$. In these papers are demonstrated that evaporation rate per drop area is inverse function of drop contact radius. The main role in the droplets evaporation is wetting of solid surface and processes in three-phase contact line gas-liquid-solid. The heat- and mass transfer processes in the contact line is important physical and technical problem which is not solved completely. The theoretical and experimental studies prove that heat transfer intensity in the contact line region can be about $1 \mathrm{~kW} / \mathrm{cm}^{2}$ [6]. Unfortunately, it is impossible to measure the heat flux in the microregion in the contact line, various non-contact methods and numerical procedures are applied. The results of the experiment with two heated vertical plates of metal foil with meniscus of HFE7100 liquid which is evaporated between them are demonstrated in [7]. It is shown that the local heat flux density in the contact line region was about 5 times higher than the average heat flux density. Heat transfer in a sessile liquid droplet was studied with numerical methods in [8]. The formation of the two vortexes surface-driven thermocapillary and volume-driven thermogravitational forces was studied. The heat flux distribution near the contact lines is investigated using the sessile drop configuration by numerical solution of the Cauchy problem for elliptic equations $[9,10,11]$. The heat

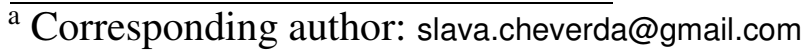


transfer in sessile drop on a thin metal foil is studied using thermochromic liquid crystals (TLCs) in [12]. The goal of this paper is to obtain heat flux distribution near the contact line using IR imaging for one and ensemble of several (3) droplets.

\section{Experimental setup and technique of measurement}

The schematic diagram of the experimental setup demonstrates in Figure 1. The basic element of the test cells is a constantan foil (CuNi) of the thickness of $25 \mu \mathrm{m}$, size of $80 \times 35 \mathrm{~mm}^{2}$ and heat conductivity $\lambda$ of $23 \mathrm{~W} / \mathrm{mK}$ was used as the substrate. This foil is soldered to the two brass electrodes (4). The substrate was heated by Joule heating from power supply (2). The temperature of the bottom foil surface was measured by IR camera Titanium 570M (7) with help of gold mirror (1). The video camera (3) is used to observe a drop (6) profile. Another video camera (5) is used to observe drop from top view. The average coefficient of heat transfer from the foil surface to surrounding air $\alpha_{\mathrm{av}}$ was found to be within $25 \mathrm{~W} / \mathrm{m}^{2} \mathrm{~K}$. These measurements were performed without droplets on the foil surface.

The heat flux on the side of IR-scanner of the foil is estimated by equation: $q_{b}=\alpha_{a v}\left(T_{w}-T_{a}\right), T_{W}$ - temperature measured by IR-scanner, $\mathrm{T}_{\mathrm{a}}$ - ambient temperature, $\alpha_{\mathrm{av}}$ - average heat transfer

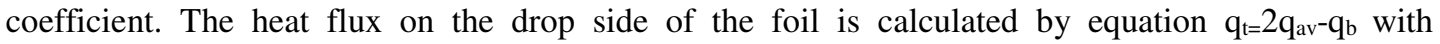
neglecting the heat conductivity along the foil, $\mathrm{q}_{\mathrm{av}}=\mathrm{P} / \mathrm{S}, \mathrm{P}$ is electrical power, $\mathrm{S}=2 \mathrm{l} \mathrm{w}$ is the total surface area of the foil.

The shape of an axisymmetric droplet lying on a flat substrate is uniquely determined by three independent geometric parameters using Nikon D300 (3). This fact allows us to calculate the physical properties, such as capillary length and contact angle, measuring three independent values, such as height and diameter of a droplet, droplet base diameter or area of its axial cross section, or its volume [13]. The average contact angle of water with constantan foil is about $60^{\circ}$. The video camera (5) is used to detect drop position on the foil.

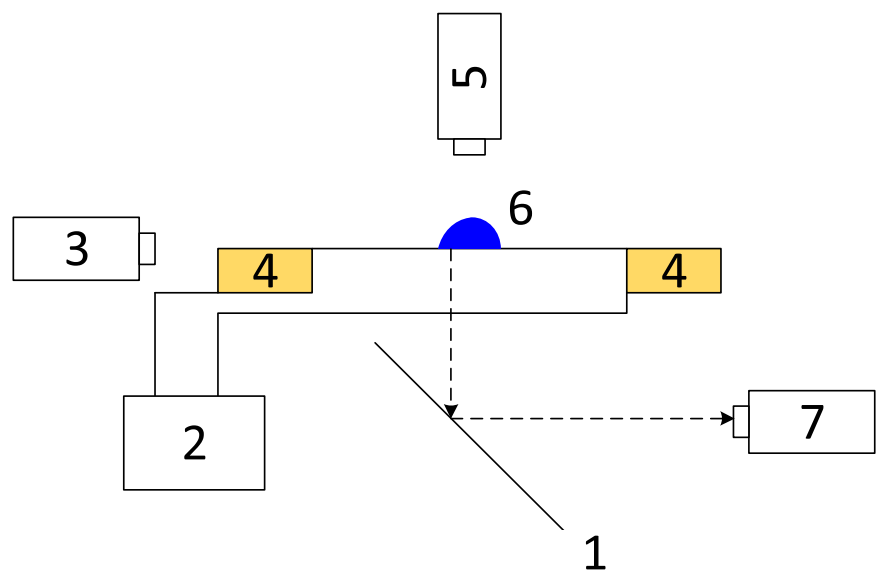

Figure 1. Schematic diagram of the experimental arrangement:

\section{Results and discussion}

Using the Cauchy problem solution, the distributions of heat flux density on the wetted foil surface have been obtained. The infrared data were used. The measured temperature field (Fig. 2 (a), Fig. 3(a), Fig. 4(a)), calculated distribution of the heat flux density on the substrate (Fig. 2 (b), Fig. 3(b), Fig. 4(b)), and heat flux density along the central drop cross section are shown in (Fig. 2 (c), Fig. 3(c), Fig. 4(c)). The results obtained for macro IR images are shown in Fig 3. 


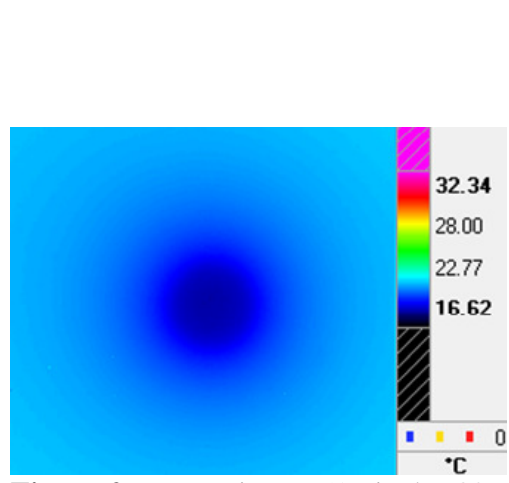

b

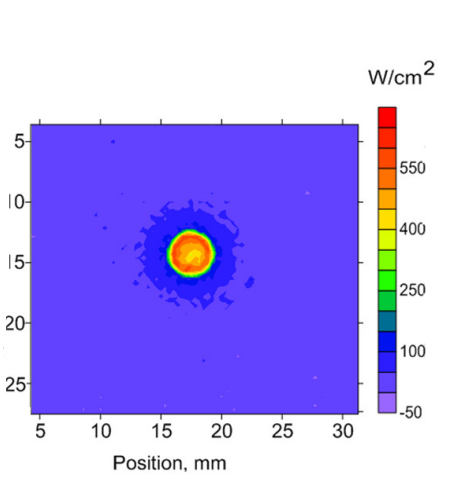

C

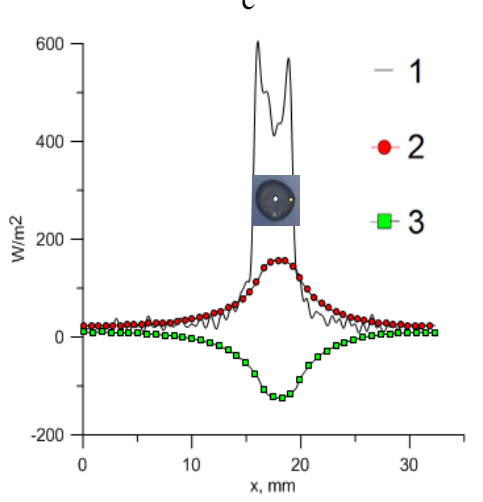

Figure 2. a - IR image (1 pixel - $83 \mu \mathrm{m}$ ) from the bottom side of the foil with drop on it (drop with diameter 3 $\mathrm{mm}$, heat flux 16. $\left.39 \mathrm{~W} / \mathrm{cm}^{2}\right), \mathrm{b}$ - heat flux distribution for Cauchy problem solution, $\mathrm{c}$ - distributions of heat flux density for the line throw the center of the drop: 1: Cauchy problem solution. 2: calculation with neglecting of heat flux redistribution in the foil, $q_{t}$. 3: on the bottom side of foil, $q_{b}$.

a

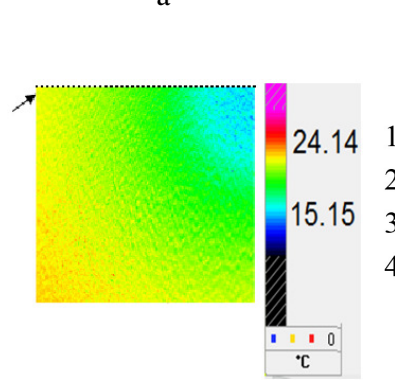

b

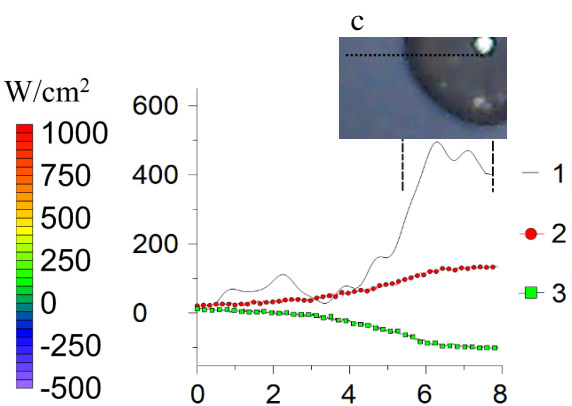

Figure 3. a - macro IR image (1 pixel $-15 \mu \mathrm{m})$ from the bottom side of the foil with drop on it (drop with diameter $3 \mathrm{~mm}$, heat flux 16. $23 \mathrm{~W} / \mathrm{cm}^{2}$ ), b - heat flux distribution for Cauchy problem solution, c - distributions of heat flux density for the line throw the center of the drop: 1: Cauchy problem solution. 2: calculation with neglecting of heat flux redistribution in the foil, $q_{t} .3$ : on the bottom side of foil, $q_{b}$.

a

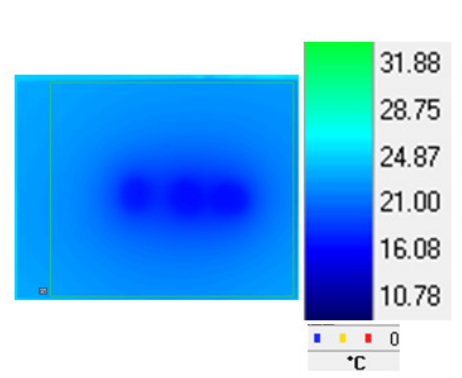

$\mathrm{b}$

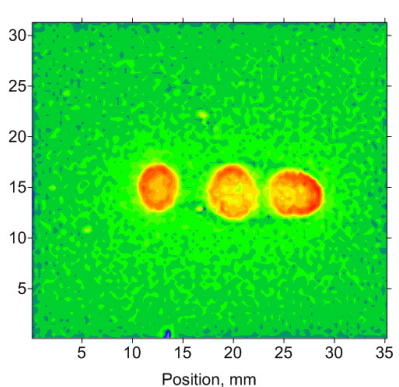

c

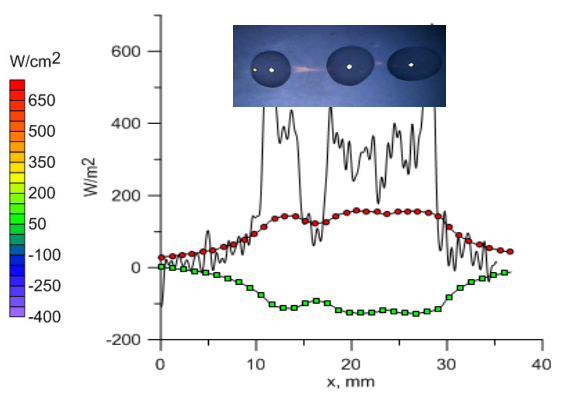

Figure 4. a - IR image ( 1 pixel $-83 \mu \mathrm{m})$ from the bottom side of the foil with three drops on it (drop with diameter $3 \mathrm{~mm}$, heat flux 16. $23 \mathrm{~W} / \mathrm{cm}^{2}$ ), b - heat flux distribution for Cauchy problem solution, c - distributions of heat flux density for the line throw the center of the drop: 1: Cauchy problem solution. 2: calculation with neglecting of heat flux redistribution in the foil, $q_{t} .3$ : on the bottom side of foil, $q_{b}$.

The results show that the maximum of the heat flux density is near the contact line and it exceeds the average heat flux density by several times. The results of measurements and calculations for three sessile drops on the substrate are shown in Figure 4. For these drops the symmetry condition is 
broken. In the contact line region between drops we do not observe the maximum of heat flux density because the heat flux from the periphery, which is now split between the two drops, decreases for each of these drops. The maximum of heat flux density is reached near the contact line at the outer edges of the three-droplet system.

Authors gratefully acknowledge support of this work by the the Russian Science Foundation (project 14-39-00003).

\section{References}

1. A. Ababneh, A. Amirfazli, and J. A. W. Elliott, Canadian Journal of Chemical Engineering, 84, 39 (2006)

2. D. Brutin, Z.-Q. Zhu, O. Rahli, J.-C. Xie, Q.-S. Liu, and L. Tadrist, Microgravity Science and Technology, 22, 387 (2010)

3. O. A. Kabov, D. V. Zaitsev, Doklady Physics, 58, 292 (2013)

4. E.Ya. Gatapova, A.A. Semenov, D.V. Zaitsev, O.A. Kabov, Colloids and Surfaces A: Physicochemical and Engineering Aspects, 441, 776 (2014)

5. A.A. Semenov, D.V. Feoktistov, D.V. Zaitsev, G.V. Kuznetsov, O.A. Kabov, Thermophysics and Aeromechanics, 22, 771 (2015)

6. P. Stephan, C. Brandt, Heat Transfer Engineering, 25, 78 (2004)

7. K. Ibrahem, M. F. Abd Rabbo, T. Gambaryan-Roisman, and P. Stephan, Experimental Thermal and Fluid Science, 34, 1036 (2010)

8. M.V. Bartashevich, I.V. Marchuk, O.A. Kabov, Thermophysics and Aeromechanics, 19, 317 (2012)

9. I. Marchuk, A. Karchevsky, A. Surtaev, O. Kabov, International Journal of Aerospace Engineering, 391036 (2015)

10. A.L. Karchevsky, Eurasian Journal of Mathematical and Computer Applications, 1, 5 (2013).

11. A.L. Karchevsky, I.V. Marchuk, O.A. Kabov, Applied Mathematical Modelling, 40, 1029 (2016)

12. C. Sodtke, V. S. Ajaev, and P. Stephan, Journal of Fluid Mechanics, 610, 343 (2008)

13. I. V. Marchuk, V. V. Cheverda, P. A. Strizhak, O. A. Kabov, Thermophysics and Aeromechanics 22, 297 (2015) 